\title{
El papel de la amilina en la función de la célula $\beta$ pancreática en pacientes con síndrome metabólico
}

\section{The role of amylin in the functionality of $\beta$-cell in patients with metabolic syndrome}

\author{
Juan Carlos Plata-Corona*, Beatriz Lara-Solís ${ }^{1}$, Ricardo PÉRez-Fuentes ${ }^{1,2}$ y EnRiQue TorRes-RasGado ${ }^{1,2}$ \\ 'Laboratorio de Investigación en Fisiopatología de las Enfermedades Crónicas (LIFEC), Unidad de Medicina Familiar n. 2, Centro de \\ Investigación Biomédica de Oriente (CIBIOR), Instituto Mexicano del Seguro Social (IMSS); ${ }^{2}$ Facultad de Medicina, Benemérita \\ Universidad Autónoma de Puebla. Puebla, México
}

\section{RESUMEN}

El objetivo principal de este estudio fue identificar si existe correlación entre los niveles plasmáticos de amilina y la funcionalidad de la célula beta ( $\beta$ ) pancreática en pacientes con síndrome metabólico (SM). Se llevó a cabo un estudio observacional, descriptivo y transversal en 283 participantes. Se determinaron variables clínicas, bioquímicas y antropométricas, y se realizó el diagnóstico de SM con base en los criterios «armonizados», clasificándose la población en $\mathrm{SM}+$ y $\mathrm{SM}$-. Se determinó el estado funcional de la célula $\beta$ mediante HOMA (Homeostatic Model Assessment) $\beta$, así como la resistencia a la insulina por medio de HOMA-IR (Insulin Resistance) e índice QUICKI (Quantitative Insulin Check Index). Se realizó el análisis estadístico, utilizando el test U de Mann-Whitney y el t de Student para las variables paramétricas y no paramétricas, se utilizó el índice de correlación de Spearman (rho) para la correlación entre la funcionalidad de la célula $\beta$ y los niveles de amilina entre ambos grupos. Se obtuvo una correlación negativa (rho: $-0.870 ; p<0.003$ ) entre la funcionalidad de la célula $\beta$ y niveles de amilina en población $\mathrm{SM}+$. En conclusión, los niveles de amilina y la función de la célula $\beta$ podrían estar relacionados en la fisiopatología del SM.

Palabras clave: Síndrome metabólico. Amilina. Célula $\beta$. Diabetes tipo 2. Índice HOMA.

\begin{abstract}
The objective of the present study was to identify the correlation between plasma levels of amylin and beta ( $\beta$ ) pancreatic cell functionality in patients with metabolic syndrome (MetS). An observational, descriptive and cross-sectional study was performed in 283 participants. Clinical, biochemical and anthropometric parameters were collected and "harmonized" criteria were used to allocate subjects to either MetS- or MetS+ group. The functional status of the $\beta$ cell was determined by HOMA (Homeostatic Model Assessment) $\beta$ and insulin resistance with HOMA-IR and QUICKI (Quantitative Insulin Check Index). Statistical analysis was performed using the Mann-Whitney $U$ test and Student's t test for parametric and nonparametric variables; Spearman's rho was used to make a correlation between $\beta$ cell function and plasmatic amylin levels. We obtained a negative correlation (rho: $-0.870, p<0.003$ ) between the functionality of the $\beta$ cell and amylin levels in the $\mathrm{SM}+$ population. In conclusion, amylin levels and $\beta$ cell function can be related in the physiopathology of MS.
\end{abstract}

Key words: Metabolic syndrome. Amylin. $\beta$ cell. Type 2 diabetes. HOMA index.

\section{Correspondencia: \\ * Juan Carlos Plata-Corona \\ E-mail: vic_plata@hotmail.com}

Fecha de recepción: 15-02-2019

Fecha de aceptación: 12-12-2019

DOI: 10.24875/RME.19001893
Disponible en internet: 25-02-2020 Rev Mex Endocrinol Metab Nutr. 2020;7:10-8

2462-4144 / ( 2019 Sociedad Mexicana de Nutricion y Endocrinologia, AC. Publicado por Permanyer. Este es un artículo open access bajo la licencia CC BY-NC-ND (http://creativecommons.org/licenses/by-nc-nd/4.0/). 
Tabla 1. Diagnóstico y componentes del SM según su definición

\begin{tabular}{|c|c|c|c|c|}
\hline & NCEP ATP III (2005) & WHO (1998) & EGIR (1999) & IDF (2005) \\
\hline Diagnóstico & $\begin{array}{l}\text { Cumplir con } 3 \text { de los } \\
\text { siguientes } 5 \text { criterios }\end{array}$ & $\begin{array}{l}\text { Evidencia de RI o DT2 } \\
\text { más } 2 \text { de los siguientes } \\
5 \text { criterios }\end{array}$ & $\begin{array}{l}\text { Hiperinsulinemia* } \\
\text { más } 2 \text { de los siguientes } \\
4 \text { criterios }\end{array}$ & $\begin{array}{l}\text { Obesidad más } 2 \text { de los } \\
\text { siguientes } 4 \text { criterios }\end{array}$ \\
\hline $\begin{array}{l}\text { Criterio } \\
\text { absolutamente } \\
\text { necesario }\end{array}$ & Ninguno & $\mathrm{RI}^{\dagger} \mathrm{o}$ DT2 & $\begin{array}{l}\text { Hiperinsulinemia (niveles } \\
\text { de insulina arriba del } \\
\text { percentil 75) }\end{array}$ & $\begin{array}{l}\text { Obesidad central } \\
\text { (perímetro de } \\
\text { cintura }): \geq 94 \mathrm{~cm} \\
(\mathrm{M}), \geq 80 \mathrm{~cm}(\mathrm{~F})\end{array}$ \\
\hline $\begin{array}{l}\text { Perímetro de } \\
\text { cintura }\end{array}$ & $\begin{array}{l}\text { Perímetro de } \\
\text { cintura } \geq 102 \mathrm{~cm}(\mathrm{M}) \\
\mathrm{y} \geq 88 \mathrm{~cm}(\mathrm{~F})\end{array}$ & $\begin{array}{l}\text { Índice cintura/ } \\
\text { cadera }>0.9(\mathrm{M}) \text { y }>0.85 \\
(\mathrm{~F}), \mathrm{o} \mathrm{IMC}>30 \mathrm{~kg} / \mathrm{m}^{2}\end{array}$ & $\begin{array}{l}\text { Perímetro de } \\
\text { cintura } \geq 94 \mathrm{~cm}(\mathrm{M}) \\
\mathrm{y} \geq 80 \mathrm{~cm}(\mathrm{~F})\end{array}$ & $\begin{array}{l}\text { Obesidad central requerida } \\
\text { necesariamente }\end{array}$ \\
\hline Hiperglucemia & $\begin{array}{l}\text { Glucosa en ayunas } \\
\geq 100 \mathrm{mg} / \mathrm{dl} \text { o en } \mathrm{Tx}\end{array}$ & $\begin{array}{l}\mathrm{RI} \text { requerida } \\
\text { necesariamente }\end{array}$ & $\begin{array}{l}\mathrm{R} \text { l requerida } \\
\text { necesariamente }\end{array}$ & $\begin{array}{l}\text { Glucosa en ayunas } \\
\geq 100 \mathrm{mg} / \mathrm{dl}\end{array}$ \\
\hline Triglicéridos & $\begin{array}{l}\text { Triglicéridos } \geq 150 \mathrm{mg} / \mathrm{dl} \\
\text { o en Tx }\end{array}$ & Triglicéridos $\geq 150$ mg/dl & Triglicéridos $\geq 177$ mg/dl & $\begin{array}{l}\text { Triglicéridos } \geq 150 \mathrm{mg} / \mathrm{dl} \\
\text { o en Tx }\end{array}$ \\
\hline Niveles de HDL & $\begin{array}{l}\mathrm{HDL}-\mathrm{C}<40 \mathrm{mg} / \mathrm{dl} \\
(\mathrm{M}),<50 \mathrm{mg} / \mathrm{dl}(\mathrm{F}), \\
\text { O en Tx }\end{array}$ & $\begin{array}{l}\mathrm{HDL}-\mathrm{C}<35 \mathrm{mg} / \mathrm{dl} \\
(\mathrm{M}),<39 \mathrm{mg} / \mathrm{dl}(\mathrm{F})\end{array}$ & $\mathrm{HDL}-\mathrm{C}<39 \mathrm{mg} / \mathrm{dl}$ & $\begin{array}{l}\mathrm{HDL}-\mathrm{C}<40 \mathrm{mg} / \mathrm{dl} \\
(\mathrm{M}),<50 \mathrm{mg} / \mathrm{dl}(\mathrm{F}), \\
O \text { en Tx }\end{array}$ \\
\hline Tensión arterial & $\begin{array}{l}>130 \text { mmHg sistólica } \\
\text { o > } 85 \text { mmHg diastólica } \\
\text { o en Tx }\end{array}$ & $\geq 140 / 90 \mathrm{mmHg}$ & $\begin{array}{l}\geq 140 / 90 \mathrm{mmHg} \\
\text { o en } \mathrm{Tx}\end{array}$ & $\begin{array}{l}>130 \text { mmHg sistólica } \\
\text { o > } 85 \text { mmHg diastólica o } \\
\text { en Tx }\end{array}$ \\
\hline Otros criterios & & Microalbuminuria ${ }^{\S}$ & & \\
\hline
\end{tabular}

*Rentable solo en pacientes sin DT2.

${ }^{\dagger}$ Medida mediante test de tolerancia a la glucosa, glucosa en ayunas, DT2 o evidencia de resistencia a la insulina mediante estudios avalados (HOMA, clamp, QUICKI).

*Valores dados para población europea.

SÍndice albuminuria/creatinina $\geq 30 \mathrm{mg} / \mathrm{g}$.

NCEP ATP III: National Cholesterol Education Program Adult Treatment Panel III; IDF: International Diabetes Federation; WHO: World Health Organization; EGIR: European Group for the Study of Insuline Resistance; M: masculino; F: femenino; RI: resistencia a la insulina; DT2: diabetes tipo 2; Tx: tratamiento farmacológico; HDL-C: colesterol HDL (lipoproteínas de alta densidad); IMC: índice de masa corporal; HOMA: Homeostatic Model Assessment; clamp: clamp euglucémico-hiperinsulinémico; QUICKI: Quantitative Insulin Check Index.

\section{INTRODUCCIÓN}

El síndrome metabólico (SM) representa una serie de alteraciones metabólicas que aparecen simultánea o consecutivamente en un individuo y que en conjunto son consideradas factores de riesgo para desarrollar diabetes tipo 2 (DT2) y enfermedades cardiovasculares $(E C V)^{1}$. Dentro de las alteraciones que lo caracterizan destacan: obesidad central, hipertensión arterial y alteraciones en el metabolismo de los glúcidos y lipoproteínas².

Según la Encuesta Nacional de Salud y Nutrición (ENSANUT) 2006, la prevalencia nacional de SM en adultos de 20 años de edad y más, de acuerdo con el National Cholesterol Education Program Adult Treatment Panel III (NCEP-ATP III), la American Heart Association (AHA) y el National, Heart, Lung and
Blood Institute (NHLBI), y la International Diabetes Federation (IDF) fue de $36.8,41.6$ y $49.8 \%$ respectivamente, siendo particularmente predominante en individuos de sexo femenino, bajo nivel sociocultural y residentes de áreas metropolitanas ${ }^{3}$.

Debido a su elevada prevalencia en la población mexicana y por el riesgo de complicaciones cardiovasculares que conlleva, representa un serio problema nacional de salud pública ${ }^{4}$.

A lo largo de los años múltiples asociaciones han postulado sus propios criterios para el diagnóstico del SM (Tabla 1). Existen algunas definiciones, como la del NCEP-ATP III, que suelen ser más utilizadas, ya que incorporan las características clave de hiperglucemia, resistencia a la insulina, obesidad visceral, dislipidemia aterogénica e hipertensión, además de que las mediciones de laboratorio que precisa están fácilmente al alcance. Es importante destacar que no requiere que 
se cumpla de forma estricta algún criterio diagnóstico. Por lo tanto, esta definición no se basa en ninguna noción preconcebida de la causa subyacente del SM, ya sea la resistencia a la insulina o la obesidad ${ }^{5}$.

Actualmente no existen criterios universales, sin embargo, y con el objetivo de homogeneizar criterios se han realizado diversos consensos. El más importante se llevó a cabo en 2009 con la participación de la IDF, la AHA y el NHLBI, logrando así unificar criterios entre estas organizaciones.

Se dieron a conocer entonces los criterios «armonizados», que fueron los utilizados en este estudio debido a que se asemejan a los establecidos por el NCEP-ATP III y la IDF con la diferencia de que proponen utilizar el perímetro de cintura ajustado a cada población; en nuestro estudio se utilizó la medida establecida para población de centro y sudamérica ${ }^{5}$.

Los criterios armonizados también puntualizan que el contar con tratamiento farmacológico para cualquier variable le confiere positividad a esta, además de que este sistema no excluye a pacientes con diagnóstico de DT2 según sus cifras de glucemia ${ }^{5,6}$.

Se requiere cumplir al menos 3 de los siguientes 5 ítems para su diagnóstico según el sistema armonizado:

1) Circunferencia de cintura: adaptado a cada población (Centro y Sudamérica $\geq 90[\mathrm{M}$, sexo masculino] $y \geq 80 \mathrm{~cm}$ [F, sexo femenino]).

2) Triglicéridos $\geq 150 \mathrm{mg} / \mathrm{dl}$ o bajo terapia farmacológica.

3) Lipoproteínas de alta densidad (HDL): $<40 \mathrm{mg} / \mathrm{dl}$ para hombres o $<50 \mathrm{mg} / \mathrm{dl}$ para mujeres o bajo terapia farmacológica.

4) Hipertensión: presión arterial sistólica $\geq 130$ $\mathrm{mmHg}$ o presión arterial diastólica $\geq 85 \mathrm{mmHg}$ o bajo terapia farmacológica

5) Glucosa en ayunas alterada: $\geq 100 \mathrm{mg} / \mathrm{dl}$ o bajo terapia farmacológica.

Una de las características principales del SM es el aumento del tejido adiposo. Este se ha relacionado con el aumento de la producción de citocinas y de ácidos grasos libres, los cuales parecen ser los responsables del desarrollo de resistencia a la insulina (RI), hiperglucemia e hiperinsulinismo para mantener la homeostasis ${ }^{7,8}$. En la última década se ha reconocido el importante papel de los adipocitos en la homeostasis de energía corporal, la sensibilidad a la insulina, y el metabolismo de carbohidratos y lípidos ${ }^{8}$.

La masa de células $\beta$ puede aumentar su número, tamaño y función como mecanismo adaptativo ante condiciones de insulinorresistencia (embarazo, obesidad, SM) y esta respuesta determina la susceptibilidad para desarrollar DT29,10. Se ha podido determinar que en pacientes obesos existe un aumento de aproximadamente de un 10 a un $30 \%$ del número de células $\beta$ por cada $10 \mathrm{~kg}$ de incremento de peso ${ }^{10}$.

La hiperinsulinemia compensatoria generada de este mecanismo adaptativo mantiene los niveles de glucemia dentro de parámetros normales, sin embargo a la par coexisten mecanismos dañinos como la glucolipotoxicidad y el estado proinflamatorio; esto produce daño a la célula $\beta$, inhibe su capacidad proliferativa y secretora, y produce finalmente muerte celular, lo que resulta en el desarrollo de intolerancia a glucosa y posteriormente DT2 $2^{9,11}$.

Se ha sugerido que al existir hiperinsulinismo aumenta también la producción de amilina, una hormona neuroendocrina peptídica cosecretada y colocalizada junto con la insulina en las células $\beta$ de los islotes pancreáticos ${ }^{12}$. Hasta la actualidad se han descrito efectos biológicos de la amilina en el musculoesquelético, el hígado, el páncreas, el sistema nervioso central, el metabolismo óseo y el sistema cardiovascular. A nivel gastrointestinal la amilina posee acciones encaminadas al control glucémico, como la inhibición del vaciamiento gástrico, la reducción del apetito y la reducción del peso corporal ${ }^{7,13}$.

Los niveles elevados de amilina causan toxicidad en la membrana de la célula $\beta$, estas acumulaciones forman pequeños ensamblajes (llamados «oligómeros») de amilina, los cuales alteran la bicapa lipídica debido a la formación de poros o canales, lo que conduce a la permeabilidad y a la pérdida de la homeostasis iónica y, por consiguiente, a una apoptosis celular, permitiendo el reemplazo de células $\beta$ por los depósitos de amiloide ${ }^{12,14-16}$.

No obstante, los mecanismos moleculares responsables del daño, en gran parte, desconocidos ${ }^{7,17}$. El 
depósito de amiloide pancreático se ha encontrado en biopsias de individuos que padecieron DT2 clínicamente establecida. Sin embargo, la amiloidosis de los islotes y la citotoxicidad celular no son características universales en los pacientes con esta enfermedad y es probable que sean solamente uno de los muchos factores que contribuyen a su desarrollo en humanos ${ }^{16}$. Se ha debatido extensamente si la presencia de depósitos de sustancia amiloide es parte de la fisiopatología del SM y la DT2, o si simplemente se trata de una

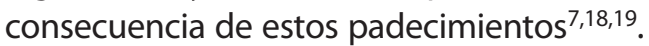

Existen diversos métodos para evaluar la funcionalidad de la célula $\beta$ y la resistencia a la insulina. Entre los distintos métodos están los invasivos, siendo el estándar de oro para la evaluación de insulinorresistencia el clamp euglucémico-hiperinsulinémico, el cual si bien es reproducible, la complejidad de su técnica lo hace poco accesible para estudios de investigación, por lo que se prefieren métodos no invasivos, los cuales han obtenido una buena correlación de los resultados al ser comparados con el estándar de oro ${ }^{20-22}$.

Estos modelos son el HOMA (Homeostatic Model Assessment) y el QUICKI (Quantitative Insulin Check Index). Según el estudio BRAMS, realizado en población brasileña, se establece que el punto de corte para HOMA-IR (Insulin Resistance) sea $\geq 2.7$, obteniendo con esto una sensibilidad del $76.8 \%$ y una especificidad del $66.7 \%$ para identificar a pacientes con $\mathrm{SM}^{23}$. El modelo HOMA posee una ventaja adicional, ya que además de determinar si existe RI (HOMA-IR) también permite valorar la funcionalidad de la célula $\beta$ (HOMA- $\beta$ ) la cual se reporta por porcentaje de células $\beta$ funcionales ${ }^{21,24}$. El índice QUICKI se basa en un modelo logarítmico que también se calcula a partir de las concentraciones de glucosa e insulina en ayuno ${ }^{22,25}$. Según el estudio de Hrebicek se encontró que existe una relación directa entre un índice QUICKI menor a 0.357 y la expresión clínica y bioquímica de las características que definen al SM, por lo cual se propone este valor como punto de corte ${ }^{26}$.

Nuestro estudio tuvo como objetivo principal determinar la correlación entre los niveles plasmáticos de amilina con el estado funcional de las células $\beta$ en pacientes con SM. Con el fin de lograr un mejor entendimiento sobre la fisiopatología del SM y de la DT2.

\section{MATERIAL Y MÉTODOS}

Se realizó un estudio observacional, descriptivo y transversal en 283 adultos entre 18 y 65 años, derechohabientes de la Unidad de Medicina Familiar n. 2 (UMF-2) del Instituto Mexicano del Seguro social (IMSS).

Se consideraron como criterios de inclusión los siguientes: derechohabientes UMF-IMSS 2, ambos sexos, edad de 18 a 65 años; mientras que los criterios de exclusión fueron: pacientes menores de 18 o mayores de 65 años, mujeres embarazadas o lactando, pacientes que una vez firmado el consentimiento informado no concluyeron con la historia clínica o la toma de muestras, pacientes que presentaron alguna otra enfermedad endocrinológica distinta a SM o DT2.

Se determinaron las siguientes variables:

- Clínicas. Edad, género :masculino o femenino, antecedentes heredofamiliares, antecedentes personales patológicos y tensión arterial; esta última se determinó por medio de la tensión arterial sistólica y la tensión arterial diastólica.

- Antropométricas. El peso y talla se calcularon utilizando un analizador de composición corporal TANITA $^{\oplus}$ (modelo TBF-215). El índice de masa corporal (IMC) se definió para cada sujeto según el valor del IMC o Índice de Quetelet (peso/talla ${ }^{2}$ ), que se expresa en $\mathrm{kg} / \mathrm{m}^{2}$ de superficie corporal. La circunferencia abdominal fue tomada con una cinta métrica y expresada en centímetros $(\mathrm{cm})$.

- Bioquímicas. Las muestras sanguíneas se recolectaron de la vena antecubital, en ayuno de 10-12 h. Se analizaron las muestras para obtener glucosa en ayuno, por medio del método de oxidación de la glucosa, los niveles plasmáticos de amilina se obtuvieron por ELISA tipo sándwich, la insulina de ayuno fue determinada por medio de radioinmunoensayo, el colesterol total, las HDL y los triglicéridos fueron enviados a un laboratorio con técnicas estandarizadas para su cálculo mediante método enzimático espectrofotométrico.

- Estado funcional y RI de la célula $\beta$. Una vez obtenidos los resultados de insulina y glucosa en 
Tabla 2. Características antropométricas de la población de estudio

\begin{tabular}{lcc}
\hline Variable & SM $(-) \mathrm{n}=83$ & $\mathrm{SM}(+) \mathrm{n}=200$ \\
\hline Edad (años) & $38.6 \pm 14.3$ & $50.8 \pm 13.6^{*}(\mathrm{p}=0.02)$ \\
\hline Sexo M/F $(\%)$ & $47 / 53$ & $40 / 60$ \\
\hline $\mathrm{IMC}\left(\mathrm{kg} / \mathrm{m}^{2}\right)$ & $23.8 \pm 3.4$ & $29.2 \pm 5.2^{*}(\mathrm{p}=0.02)$ \\
\hline Perímetro de cintura masculino $(\mathrm{cm})$ & $85.7 \pm 9.7$ & $99.4 \pm 9.4^{*}(\mathrm{p}=0.01)$ \\
\hline Perímetro de cintura femenino $(\mathrm{cm})$ & $82.2 \pm 91$ & $95.9 \pm 12.2^{*}(\mathrm{p}=0.02)$ \\
\hline Tensión arterial sistólica $(\mathrm{mmHg})$ & $111.5 \pm 10.1$ & $119.3 \pm 22.1^{\dagger}(\mathrm{p}=0.003)$ \\
\hline Tensión arterial diastólica $(\mathrm{mmHg})$ & $73.9 \pm 7.7$ & $77.4 \pm 18.9^{\dagger}(\mathrm{p}=0.002)$ \\
\hline
\end{tabular}

Los valores se expresan en medias \pm desviación estándar.

${ }^{*} \mathrm{p}<0.05$ con $\mathrm{t}$ de Student.

${ }^{\dagger} \mathrm{p}<0.05$ con $U$ de Mann-Whitney.

SM: síndrome metabólico; M: sexo masculino; F: sexo femenino; IMC: índice de masa corporal.

ayunas se evaluó la RI y la funcionalidad de la célula $\beta$ por medio de los siguientes modelos:

- HOMA- $\beta=20 \times$ insulina en ayunas $(\mu \mathrm{Ul} / \mathrm{ml}) /$ (glucosa en ayunas $[\mathrm{mmol} / \mathrm{l}]-3,5$ )

- HOMA-IR = insulina en ayunas $(\mu \mathrm{Ul} / \mathrm{ml}) \times$ glucosa en ayunas $(\mathrm{mmol} / \mathrm{l}) / 22.5$

- QUICKI = 1/[(log insulina plasmática en ayuno $(\mu \mathrm{U} / \mathrm{ml})+$ log glucosa plasmática en ayuno $(\mathrm{mg} / \mathrm{dl})]$

- Diagnóstico de SM. Se realizó con base en los criterios armonizados del 2009 considerándose como positivo $(\mathrm{SM}+)$ a los pacientes con $\geq 3$ criterios positivos y como negativo (SM-) a los pacientes con $<3$ criterios positivos.

\section{Análisis estadístico}

Se utilizó el programa estadístico SPSS ${ }^{\circledR}$ (Statistical Package for the Social Sciences) versión 20.0. Se empleó un análisis estadístico incluyendo análisis de correlación de acuerdo con los objetivos planteados y los resultados obtenidos. Se utilizaron los test $U$ de Mann-Whitney para las variables no paramétricas, las cuales fueron únicamente el nivel de triglicéridos y tensión arterial, y la t de Student para el resto de las variables entre dos grupos. Se analizaron los niveles plasmáticos de amilina y de la función de la célula $\beta$ según tuviese o no el diagnóstico de SM. Se calculó el promedio, la desviación estándar y los rangos de las variables estudiadas comparándolos entre los dos grupos de estudio. Al obtener los resultados estadísticos se realizó una correlación de dichos resultados mediante rho de Spearman en busca de la existencia de significancia estadística y la relación entre estas variables.

\section{RESULTADOS}

Del $100 \%(n=283)$ de los sujetos incluidos en el estudio, el $71 \%(n=200)$ presentaron más de tres factores para SM y esto permitió categorizarlos como $\mathrm{SM}+$. Entre los pacientes diagnosticados de $S M$, el $40 \%(n=80)$ eran pacientes con DT2, y la mitad de estos ya tomaba medicamentos para regular su glucemia (sulfonilureas, biguanidas, meglitinidas y agonistas del receptor GLP-1). La distribución del sexo fue: 164 mujeres y 119 varones en la población general. Del total de mujeres, 120 (73\%) fueron diagnosticadas de SM, mientras que 80 varones (67.2\%) fueron diagnosticados de SM.

Las variables antropométricas y metabólicas de nuestra población se describen en las siguientes tablas según sean SM+ o SM- (Tablas 2 y 3), los resultados se expresan en media y desviación estándar, además se señalan las variables con diferenciaestadísticamente significativa entre ambas poblaciones.

Se encontraron alteraciones significativas respecto a las variables bioquímicas en los pacientes con $\mathrm{SM}+$, se evidenció un aumento de los niveles de glucemia, triglicéridos e insulina, y una disminución 
Tabla 3. Características metabólicas de la población de estudio

\begin{tabular}{lcc}
\hline Variable & SM $(-) \mathrm{n}=83$ & $\mathrm{SM}(+) \mathrm{n}=200$ \\
\hline Glucosa en ayuno $(\mathrm{mg} / \mathrm{dl})$ & $93.2 \pm 11.8$ & $141 \pm 65.0^{*}(\mathrm{p}=0.003)$ \\
\hline Insulina $(\mu \mathrm{U} / \mathrm{ml})$ & $7.6 \pm 4.0$ & $11.26 \pm 6.02^{*}(\mathrm{p}=0.004)$ \\
\hline Triglicéridos $(\mathrm{mg} / \mathrm{dl})$ & $114.2 \pm 54.3$ & $218.5 \pm 69.7^{\dagger}(\mathrm{p}=0.001)$ \\
\hline $\mathrm{HDL}(\mathrm{mg} / \mathrm{dl})$ & $36.1 \pm 19.1 \mathrm{M}: 32.8 / \mathrm{F}: 39.5$ & $22.6 \pm 12.6^{*}(\mathrm{p}=0.01)$ \\
& & M: 19.4/F: 25.9 \\
\hline
\end{tabular}

Los valores se expresan en medias y desviación estándar.

${ }^{*} p<0.05$ con $t$ de Student.

${ }^{t} \mathrm{p}<0.05$ con $U$ de Mann-Whitney.

SM: síndrome metabólico; M: sexo masculino, F: sexo femenino; HDL: lipoproteínas de alta densidad.

Fuente: Laboratorio de Investigación en Fisiopatología de las Enfermedades Crónicas, Centro de Investigación Biomédica de Oriente, Unidad Medicina Familiar n. 2 , Instituto Mexicano del Seguro Social.

Tabla 4. Índice de resistencia a la insulina

\begin{tabular}{lcc}
\hline Variable & $\mathrm{SM}(-) \mathrm{n}=83$ & $\mathrm{SM}(+) \mathrm{n}=200$ \\
\hline HOMA-IR & $1.8 \pm 0.9$ & $3.8 \pm 0.5^{*}(p=0.005)$ \\
\hline QUICKI & $0.36 \pm 0.02$ & $0.32 \pm 0.02^{\dagger}(p=0.03)$ \\
\hline
\end{tabular}

Los datos se presentan como medias \pm desviación estándar.

${ }^{*} p<0.05$ con t de Student.

${ }^{+} p<0.05$ con $U$ de Mann-Whitney.

HOMA-IR: Homeostatic Model Assessment-Insulin Resistance; QUICKI: Quantitative Insulin Check Index.

Fuente: Laboratorio de Investigación en Fisiopatología de las Enfermedades

Crónicas, Centro de Investigación Biomédica de Oriente, Unidad Medicina Familiar n. ${ }^{\circ}$, Instituto Mexicano del Seguro Social.

de los niveles de HDL tanto en hombres como mujeres en esta población.

El grupo con $\mathrm{SM}+$ presentó valores significativamente mayores de HOMA-IR comparado con el grupo SM- (3.8 \pm 0.5 vs. $1.8 \pm 0.9)$. Por otra parte, los pacientes con $\mathrm{SM}+$ presentaron significativamente una disminución del índice QUICKI vs. los SM$(0.32 \pm 0.02$ vs. $0.36 \pm 0.02)$ (Tabla 4).

La alteración de estos parámetros, asociada al estado de hiperinsulinemia que se evidenció en las variables bioquímicas, confirma el estado de insulinorresistencia en el cual se encuentran los pacientes con SM. Se aprecia que en los pacientes SM+ el índice HOMA-IR y el índice QUICKI superan los puntos de corte previamente mencionados, mientras que los pacientes SM- se encuentran dentro de la normalidad.

Los pacientes $\mathrm{SM}+$ presentaron una disminución significativa de la función de la célula $\beta$ comparado con los pacientes SM- (44.7.7 \pm 5.7 vs. $75.6 \pm 9.0$; $\mathrm{p}<0.05$ ) (Fig. 1).

Nuestros resultados muestran que los pacientes con $\mathrm{SM}+$ presentaron valores significativamente más altos de amilina que los pacientes SM- $(9.9 \pm 0.6 \mathrm{vs}$. $7.8 \pm 0.5, \mathrm{pmol} / \mathrm{l} ; \mathrm{p}<0.05$ ) (Fig. 2). Como ya mencionamos, la amilina es cosecretada con la insulina, por lo cual es lógico pensar que ante el estado hiperinsulinémico de los pacientes $\mathrm{SM}+$, existe también un estado de hiperamilinemia.

Finalmente estudiamos la correlación entre la funcionalidad de la célula $\beta$ y la amilina. En la población SM- se observó una correlación prácticamente nula entre el HOMA- $\beta$ y la amilina (rho: 0.150; $p<0.07$ ). Sin embargo, al analizar esta correlación de acuerdo con la presencia de SM se observó que existe una correlación negativa (rho: $-0.870 ; p<0.003$ ) (Fig. 3).

La correlación negativa resalta el hecho de que a mayores niveles de amilina existe menor funcionalidad de la célula $\beta$ pancreática, lo cual sugiere participación de este péptido en la disfunción celular favoreciendo el desarrollo de DT2.

\section{DISCUSIÓN}

En nuestro estudio encontramos que la prevalencia de SM en nuestra población fue del $71 \%$, mientras que en el mundo apenas se alcanza un $25 \%$. Un motivo de tal discrepancia es que el diagnóstico de SM se realizó con criterios armonizados del $2009^{27}$, 


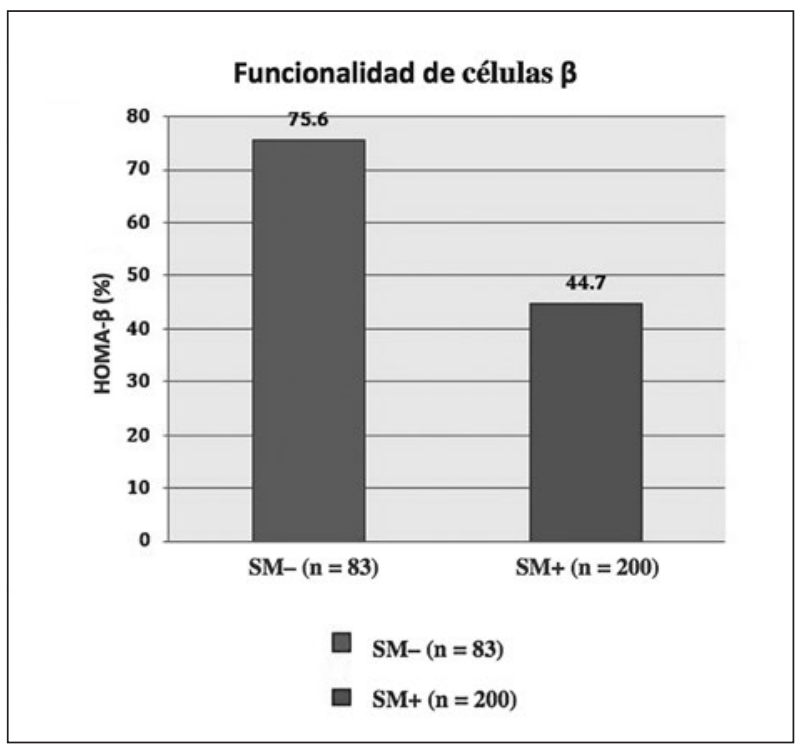

Figura 1. Determinación de la función de la célula $\beta$ por el índice HOMA- $\beta$. Se observa una reducción de la funcionalidad de células $\beta$ en pacientes $\mathrm{SM}+$, mientras que los SM- conservan aún adecuada funcionalidad. HOMA-ß: Homeostatic Model Assessment-células beta; SM: síndrome metabólico.

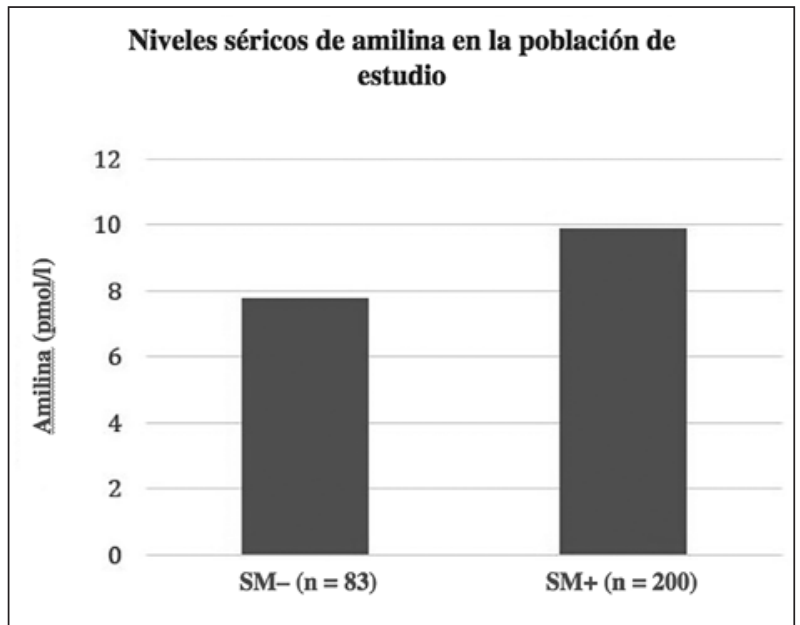

Figura 2. Niveles séricos de amilina en la población de estudio. Observamos un aumento de la secreción de amilina en los pacientes $\mathrm{SM}+$, y menores cantidades de amilina en los SM-. Los datos se expresan en media \pm desviación estándar (fuente: Laboratorio de Investigación en Fisiopatología de las Enfermedades Crónicas, Centro de Investigación Biomédica de Oriente, Unidad Medicina Familiar n.o 2, Instituto Mexicano del Seguro Social). ${ }^{*} \mathrm{p}<0.05$ con U de MannWhitney. SM: síndrome metabólico.

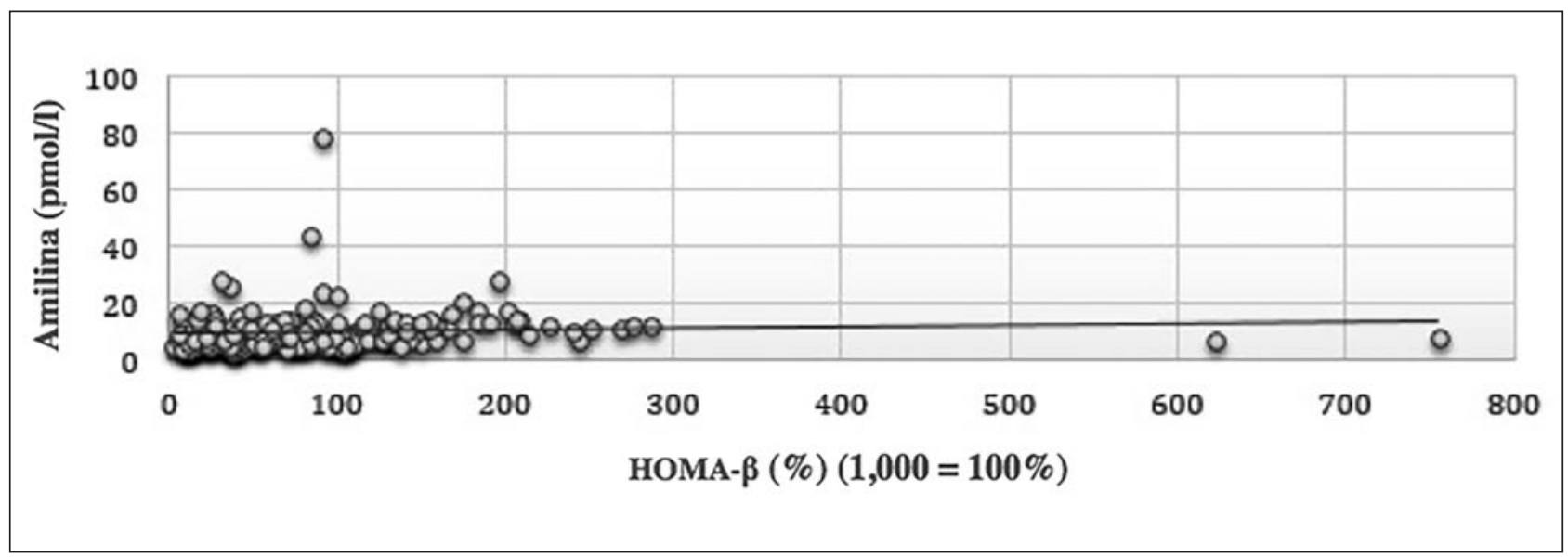

Figura 3. Correlación de la funcionalidad de la célula $\beta($ HOMA- $\beta$ ) y niveles de amilina en población SM+. Se observa que existe una correlación negativa, ya que a mayor disminución de la funcionalidad existe mayor cantidad de amilina en pacientes con SM+ (fuente: Laboratorio de Investigación en Fisiopatología de las Enfermedades Crónicas, Centro de Investigación Biomédica de Oriente, Unidad Medicina Familiar n. ${ }^{\circ}$, Instituto Mexicano del Seguro Social). HOMA-ß;: Homeostatic Model Assessment-células beta; SM: síndrome metabólico.

a diferencia de otros estudios, los cuales se rigen por los criterios de la Organización Mundial de la Salud para la realización del diagnóstico ${ }^{28}$.

Considerando que el SM se caracteriza por presentar alteraciones en el metabolismo de los hidratos de carbono (aumento de glucosa $>100 \mathrm{mg} / \mathrm{dl}$ ) y los lípidos (triglicéridos $>150 \mathrm{mg} / \mathrm{dl}, \mathrm{HDL}<40 \mathrm{mg} / \mathrm{dl}$ en hombres $\mathrm{y}<50 \mathrm{mg} / \mathrm{dl}$ en mujeres), nuestro estudio mostró que los pacientes con $\mathrm{SM}+$ presentan alteraciones metabólicas en comparación con los SM-. Este mismo comportamiento se ha observado en estudios previos ${ }^{29-31}$.

Encontramos también un aumento significativo de la insulina, con lo que confirmamos el estado hiperinsulinémico de los pacientes con $\mathrm{SM}+$, los mismos hallazgos que fueron descritos por otros autores que obtuvieron resultados similares 32,33 . 
Además del estado hiperfuncionante secundario al estado de insulinorresistencia, debemos mencionar que el aumento de los niveles de insulina en nuestra población podría ser a su vez potenciado por el uso de medicamentos secretagogos de insulina en la población $\mathrm{SM}+$, efecto que ya se ha corroborado principalmente en los pacientes bajo tratamiento con sulfonilureas.

El índice HOMA-IR arrojó resultados claramente más elevados en la población con SM+, el índice QUICKI arrojó valores disminuidos, deduciendo así que los pacientes con $\mathrm{SM}+$ poseen diferentes grados de $\mathrm{R}^{34}$. Estudio que se corrobora por Báez-Duarte, donde se mostró que los sujetos del grupo con SM comparados con el grupo de control están en un estado de insulinorresistencia ${ }^{24,35}$.

Tanto el HOMA-IR como el QUICKI han mostrado en diversos estudios que guardan relación con el número de componentes del SM. De este modo, el HOMA-IR muestra valores más elevados conforme aumentan el número de componentes de $S M$ y el QUICKI muestra valores más disminuidos a razón del aumento de componentes del SM. El índice HOMA- $\beta$ no ha demostrado guardar correlación alguna con el número de componentes del $\mathrm{SM}^{36}$.

Según artículos, en pacientes con SM y en individuos obesos las concentraciones plasmáticas de amilina suelen estar incrementadas; misma situación se encuentra en nuestro estudio donde se aprecian niveles de amilina mayores en pacientes con $\mathrm{SM}+$ en contraste con los SM- $(9.9 \pm 0.6$ vs. $7.8 \pm 0.5$, pmol/l; $\mathrm{p}<0.05)^{32,37-39}$.

En nuestra población se evaluó la funcionalidad de la célula $\beta$ mediante el índice HOMA- $\beta$, encontramos que existe una disminución de la funcionalidad en los pacientes con SM+ en comparación con los SM- (44.7.7 \pm 5.7 vs. $75.6 \pm 9.0 ; p<0.05)$, usualmente la tendencia reportada sobre la funcionalidad de la célula $\beta$ es el aumento de esta debido a la compensación metabólica ${ }^{36}$.

Sin embargo algunos estudios han considerado una variación dinámica de la funcionalidad de la célula $\beta$, observándose que a mayor tiempo de evolución existe mayor disminución de la funcionalidad ${ }^{40}$.

Al analizar la correlación entre los niveles de amilina y la funcionalidad de la célula $\beta$, encontramos que existe una relación negativa, ya que a mayores niveles de amilina existe menor funcionalidad de célula $\beta$; proponemos entonces que existe una participación de la amilina en el deterioro de la función pancreática y el desarrollo de DT2.

Los autores Juan Carlos Plata Corona y Beatriz Lara Solís colaboraron en misma proporción para el desarrollo del presente artículo.

\section{FINANCIAMIENTO}

Financiado por los mismos autores.

\section{CONFLICTO DE INTERESES}

Los autores declaran no tener conflicto de intereses.

\section{RESPONSABILIDADES ÉTICAS}

Protección de personas y animales. Los autores declaran que para esta investigación no se han realizado experimentos en seres humanos ni en animales.

Confidencialidad de los datos. Los autores declaran que han seguido los protocolos de su centro de trabajo sobre la publicación de datos de pacientes.

Derecho a la privacidad y consentimiento informado. Los autores han obtenido el consentimiento informado de los pacientes y/o sujetos referidos en el artículo. Este documento obra en poder del autor de correspondencia.

\section{BIBLIOGRAFÍA}

1. Pereira Rodríguez J, Melo Ascanio J, Caballero Chavarro M, Rincón González G, Jaimes Martín T, Niño Serrato R. Síndrome metabólico. Apuntes de interés. 2016;22(2):9.

2. Alegría Ezquerra E, Castellano Vázquez JM, Alegría Barrero A. Obesidad, síndrome metabólico y diabetes: implicaciones cardiovasculares y actuación terapéutica. Rev Esp Cardiol. 2008;61(7):752-64. 
3. Rojas R, Aguilar-Salinas CA, Jiménez-Corona A, Shamah-Levy T, Rauda J, Ávila-Burgos $\mathrm{L}$, et al. Metabolic syndrome in Mexican adults: results from the National Health and Nutrition Survey 2006. Salud pública Méx. 2010;52(Suppl 1):S11-8.

4. Lizarzaburu Robles JC. Síndrome metabólico: concepto y aplicación práctica. An Fac med. 2013;74(4):315-20.

5. Huang PL. A comprehensive definition for metabolic syndrome. Dis Model Mech. 2009;2(5-6):231-7.

6. Porchia LM, González-Mejía ME, Torres-Rasgado E, Ruiz-Vivanco G, PérezFuentes R. Low serum uric acid concentration augments insulin effects on the prevalence of metabolic syndrome. Diabetes Metab Syndr. 2018;12(3):325-31.

7. Rojas I, Novials A. Amilina: del estudio molecular a las acciones fisiológicas. Endocrinol nutr. 2001;48(8):234-45.

8. Torres DG. Tejido adiposo como glándula endocrina. Implicaciones fisiopatológicas. Revista Finlay. 2011;1(2).

9. Prentki M, Nolan CJ. Islet beta cell failure in type 2 diabetes. J Clin Invest. 2006;116(7):1802-12.

10. Yoon $\mathrm{KH}$, Ko SH, Cho JH, Lee JM, Ahn YB, Song KH, et al. Selective betacell loss and alpha-cell expansion in patients with type 2 diabetes meIlitus in Korea. J Clin Endocrinol Metab. 2003;88(5):2300-8.

11. Alejandro EU, Gregg B, Blandino-Rosano M, Cras-Meneur C, Bernal-Mizrachi $\mathrm{E}$. Natural history of beta-cell adaptation and failure in type 2 diabetes. Mol Aspects Med. 2015;42:19-41.

12. Divakara MB, Martinez D, Ravi A, Bhavana V, Ramana V, Habenstein B, et al. Molecular mechanisms for the destabilization of model membranes by islet amyloid polypeptide. Biophys Chem. 2019;245:34-40.

13. Watve $M$, Bodas $A$, Diwekar M. Altered autonomic inputs as a cause of pancreatic $\beta$-cell amyloid. Med Hypotheses. 2014;82(1):49-53.

14. Clark A, Wells CA, Buley ID, Cruickshank JK, Vanhegan RI, Matthews DR, et al. Islet amyloid, increased A-cells, reduced B-cells and exocrine fibrosis: quantitative changes in the pancreas in type 2 diabetes. Diabetes Res. 1988:9(4):151-9.

15. Lehto S, Ronnemaa T, Pyorala K, Laakso M. Cardiovascular risk factors clustering with endogenous hyperinsulinaemia predict death from coronary heart disease in patients with Type II diabetes. Diabetologia. 2000;43(2):148-55.

16. Raleigh D, Zhang X, Hastoy B, Clark A. The $\beta$-cell assassin: IAPP cytotoxicity. J Mol Endocrinol. 2017;59(3):R121-R140.

17. Sasahara K, Morigaki K, Shinya K. Amyloid aggregation and deposition of human islet amyloid polypeptide at membrane interfaces. FEBS J. 2014;281(11):2597-612.

18. Kanatsuka A, Kou S, Makino H. IAPP/amylin and beta-cell failure: implication of the risk factors of type 2 diabetes. Diabetol Int. 2018;9(3):143-57.

19. Blackard WG, Clore JN, Kellum JM. Amylin/insulin secretory ratios in morbidly obese man: inverse relationship with glucose disappearance rate. J Clin Endocrinol Metab. 1994;78(5):1257-60.

20. Okita K, Iwahashi H, Kozawa J, Okauchi Y, Funahashi T, Imagawa A, et al. Homeostasis model assessment of insulin resistance for evaluating insulin sensitivity in patients with type 2 diabetes on insulin therapy. Endocr J. 2013;60(3):283-90.

21. Reyes-Muñoz E, Martínez-Herrera E, Ortega-González C, Arce-Sánchez L, Ávila-Carrasco A, Zamora-Escudero R. Valores de referencia de HOMA-IR y QUICKI durante el embarazo en mujeres mexicanas. Ginecol Obstet Mex. 2017;85:306-13.

22. Holzinger U, Kitzberger R, Fuhrmann V, Funk GC, Madl C, Ratheiser K. Correlation of calculated indices of insulin resistance (QUICKI and HOMA) with the euglycaemic hyperinsulinaemic clamp technique for evaluating insulin resistance in critically ill patients. Eur J Anaesthesiol. 2007;24(11):966-70.

23. Geloneze B, Vasques AC, Stabe CF, Pareja JC, Rosado LE, Queiroz EC, et al. HOMA1-IR and HOMA2-IR indexes in identifying insulin resistance and metabolic syndrome: Brazilian Metabolic Syndrome Study (BRAMS) Arquivos brasileiros de endocrinologia e metabologia. 2009;53(2):281-7.

24. Garg MK, Dutta MK, Mahalle N. Study of beta-cell function (by HOMA model) in metabolic syndrome. Indian J Endocrinol Metab. 2011;15(Supp 1):S44-S9.

25. Otten J, Ahrén B, Olsson T. Surrogate measures of insulin sensitivity vs the hyperinsulinaemic-euglycaemic clamp: a meta-analysis. Diabetologia. 2014;57(9):1781-8.

26. Hrebicek J, Janout V, Malincikova J, Horakova D, Cizek L. Detection of insulin resistance by simple quantitative insulin sensitivity check index QUICKI for epidemiological assessment and prevention. J Clin Endocrinol Metab. 2002;87(1):144-7.

27. Zimmet P MAK, Serrano Ríos M. A new International Diabetes Federation worldwide definition of the metabolic syndrome: the rationale and the results. Rev Esp Cardiol (English Edition). 2005:58(12):1371-5.

28. Fernández-Bergés D, Félix-Redondo FJ, Lozano L, Pérez-Castán JF, Sanz $\mathrm{H}$, Cabrera de León $\mathrm{A}$, et al. Prevalencia de síndrome metabólico según las nuevas recomendaciones de la OMS: Estudio HERMEX. Gac Sanit. 2011;25:519-24.

29. Gluvic Z, Zaric B, Resanovic I, Obradovic M, Mitrovic A, Radak D, et al Link between metabolic syndrome and insulin resistance. Curr Vasc Pharmacol. 2017;15(1):30-9.

30. Cho J, Hong H, Park S, Kim S, Kang H. Insulin resistance and its association with metabolic syndrome in Korean children. Biomed Res Int 2017:2017:7.

31. Galarreta Aima Cl, Donet Mostacero JA, Huamán Saavedra JJ. Síndrome metabólico en la población adulta de Trujillo de acuerdo a diferentes definiciones. Acta Méd Peruana. 2009:26:217-25.

32. Gonzalez-Mejia ME, Porchia LM, Torres-Rasgado E, Ruiz-Vivanco G, Pulido-Perez P, Baez-Duarte BG, et al. C-Peptide Is a Sensitive Indicator for the Diagnosis of Metabolic Syndrome in Subjects from Central Mexico. Metab Syndr Relat Disord. 2016;14(4):210-6.

33. Costa B, Cabré JJ, Martín F. Síndrome metabólico, resistencia a la insulina y diabetes. ¿Qué se oculta bajo la punta del iceberg? Aten Prim 2003;31(7):436-45.

34. Matthews DR, Hosker JP, Rudenski AS, Naylor BA, Treacher DF, Turner RC. Homeostasis model assessment: insulin resistance and $\beta$-cell function from fasting plasma glucose and insulin concentrations in man. Diabetologia. 1985;28(7):412-9.

35. Baez-Duarte BG, Sánchez-Guillén MDC, Pérez-Fuentes R, Zamora-Ginez I, Leon-Chavez BA, Revilla-Monsalve $C$, et al. $\beta$-cell function is associated with metabolic syndrome in Mexican subjects. Diabetes Metab Syndr Obes. 2010;3:301-9.

36. Udenze I, Taiwo I, Minari J, Amadi C. Markers of insulin resistance and their performance in adult Nigerians with metabolic syndrome. Int $J$ Noncommun Dis. 2019;4(2):43-8.

37. Baez-Duarte BG, Zamora-Ginez I, De Jesus KL, Torres-Rasgado E, Gonzalez-Mejia ME, Porchia L, et al. Association of the metabolic syndrome with antioxidant defense and outstanding superoxide dismutase activity in Mexican subjects. Metab Syndr Relat Disord. 2016;14(3):154-60.

38. Eriksson J, Nakazato M, Miyazato M, Shiomi K, Matsukura S, Groop L. Islet amyloid polypeptide plasma concentrations in individuals at increased risk of developing type 2 (non-insulin-dependent) diabetes mellitus. Diabetologia. 1992;35(3):291-3.

39. Malin SK, Kirwan JP. Fasting hyperglycaemia blunts the reversal of impaired glucose tolerance after exercise training in obese older adults. Diabetes Obes Metab. 2012;14(9):835-41.

40. Cai X, Xia L, Pan Y, He D, Zhu H, Wei T, et al. Differential role of insulin resistance and $\beta$-cell function in the development of prediabetes and diabetes in middle-aged and elderly Chinese population. Diabetol Metab Syndr. 2019;11(1):24. 